\title{
On Mann's Type Method for Nonexpansive and Strongly Quasinonexpansive Mappings in Hilbert Spaces
}

\author{
Nawab Hussain, ${ }^{1}$ Giuseppe Marino, ${ }^{1,2}$ and Badriah A. S. Alamri ${ }^{1}$ \\ ${ }^{1}$ Department of Mathematics, King Abdulaziz University, P.O. Box 80203, Jeddah 21589, Saudi Arabia \\ ${ }^{2}$ Dipartimento di Matematica, Universitá della Calabria, Arcavacata, 87036 Rende, Italy \\ Correspondence should be addressed to Giuseppe Marino; giuseppe.marino@unical.it
}

Received 3 February 2015; Accepted 24 April 2015

Academic Editor: Adrian Petrusel

Copyright (c) 2015 Nawab Hussain et al. This is an open access article distributed under the Creative Commons Attribution License, which permits unrestricted use, distribution, and reproduction in any medium, provided the original work is properly cited.

In the setting of Hilbert spaces, we study Mann's type method to approximate strong solutions of variational inequalities. We show that these solutions are fixed points of a nonexpansive mapping and/or a strongly quasinonexpansive mapping, depending on the coefficients involved in the algorithm.

\section{Introduction}

Let $H$ be a real Hilbert space with inner product $\langle\cdot, \cdot\rangle$ and induce a norm $\|\cdot\|$. Let $T: H \rightarrow H$ be a nonlinear mapping. $\operatorname{Fix}(T):=\{x \in H: x=T x\}$ denotes the fixed point set of $T$.

The problem of approximating fixed points of nonlinear mapping has been widely investigated by many authors.

The starting point of the present paper is the following scheme introduced by Iemoto and Takahashi in [1]:

$$
x_{n+1}=\left(1-\alpha_{n}\right) x_{n}+\alpha_{n}\left[\beta_{n} T x_{n}+\left(1-\beta_{n}\right) S x_{n}\right] \text {, }
$$

where $T$ is a nonexpansive mapping and $S$ is a nonspreading mapping.

Depending on the coefficients $\alpha_{n}$ and $\beta_{n}$, in [1] was obtained weak convergence of the method either to a fixed point of $T$ or to a fixed point of $S$ or to a common fixed point of $T$ and $S$.

Motivated by this result, with the aim of obtaining strong convergence, in [2] we introduced a viscosity in a previous scheme and we have been able to prove the following result, in which the most important role was played by the average type mappings $T_{\delta}=(1-\delta) I+\delta T$ and $S_{\delta}=(1-\delta) I+\delta S$, $\delta \in(0,1)$.
Theorem 1 (see [2]). Let $T: H \rightarrow H$ be a nonexpansive mapping and let $S: H \rightarrow H$ be a nonspreading mapping. Let

$$
\begin{aligned}
x_{1} & \in C, \\
x_{n+1} & =\alpha_{n} f\left(x_{n}\right)+\left(1-\alpha_{n}\right)\left[\beta_{n} T_{\omega} x_{n}+\left(1-\beta_{n}\right) S_{\omega} x_{n}\right], \\
n \geq 1, &
\end{aligned}
$$

with $\left(\alpha_{n}\right)_{n} \subset(0,1), \alpha_{n} \rightarrow 0$, and $\sum_{n} \alpha_{n}=\infty$. Then

(i) if $\sum_{n}\left(1-\beta_{n}\right)<\infty, \sum_{n}\left|\alpha_{n}-\alpha_{n+1}\right|<\infty$, and $\operatorname{Fix}(T) \neq \emptyset$, then $\left(x_{n}\right)$ strongly converges to $\bar{p}=P_{\mathrm{Fix}(T)} f(\bar{p})$ which is the unique point in $\operatorname{Fix}(T)$ that satisfies the variational inequality

$$
\langle(I-f) \bar{p}, x-\bar{p}\rangle \geq 0, \quad \forall x \in \operatorname{Fix}(T) ;
$$

(ii) if $\sum_{n} \beta_{n}<\infty$ and $\operatorname{Fix}(S) \neq \emptyset$, then $\left(x_{n}\right)_{n}$ converges strongly to $\widetilde{p}=P_{\operatorname{Fix}(S)} f(\widetilde{p})$ which is the unique point in $\operatorname{Fix}(S)$ that satisfies the variational inequality

$$
\langle(I-f) \widetilde{p}, x-\widetilde{p}\rangle \geq 0, \quad \forall x \in \operatorname{Fix}(S) ;
$$

(iii) if $\liminf _{n} \beta_{n}\left(1-\beta_{n}\right)>0$ and $\operatorname{Fix}(T) \cap \operatorname{Fix}(S) \neq \emptyset$, then $\left(x_{n}\right)$ strongly converges to $p_{0}=P_{\mathrm{Fix}(T) \cap \mathrm{Fix}(S)} f\left(p_{0}\right)$ 
which is the unique point in $\operatorname{Fix}(T) \cap \operatorname{Fix}(S)$ that satisfies the variational inequality

$\left\langle(I-f) p_{0}, x-p_{0}\right\rangle \geq 0, \quad \forall x \in \operatorname{Fix}(T) \cap \operatorname{Fix}(S)$.

At this point, three questions can be posed:

(1) Is it possible to replace the coefficient $\left(1-\alpha_{n}\right)$ with the term $\left(I-\alpha_{n} A\right)$ where $A: H \rightarrow H$ is a linear, bounded, strongly positive operator? In doing so, we would be able to solve some minimization problem.

(2) Is it possible to replace the type average mappings $T_{\delta}$ and $S_{\delta}$ with the original mappings $T$ and $S$ ?

(3) Is it possible to replace the nonspreading mapping $S$ with a more general type of mappings?

We will see here that the answers are all positive.

A natural class of mappings containing the nonspreading mappings is given by the class of hybrid mappings. Following Aoyama et al. [3] we say that $S: H \rightarrow H$ is an $L$-hybrid mapping, with $L \geq 0$, if

$$
\begin{aligned}
\|S x-S y\|^{2} \leq\|x-y\|^{2}+L\langle x-S x, y-S y\rangle & \\
& \forall x, y \in H
\end{aligned}
$$

or equivalently

$$
\begin{aligned}
2\|S x-S y\|^{2} \leq & \|x-S y\|^{2}+\|y-S x\|^{2} \\
& -2\left(1-\frac{L}{2}\right)\langle x-S x, y-S y\rangle .
\end{aligned}
$$

We denote by $\mathscr{H}_{L}$ the family of $L$-hybrid mappings defined on the space $H$.

Note that particular choices of $L$ give important families of nonlinear mappings:

(i) $\mathscr{H}_{0}$ is the family of nonexpansive mappings (i.e., $\| S x-$ $S y\|\leq\| x, y \|$ for all $x, y \in H)$.

(ii) $\mathscr{H}_{2}$ is the family of nonspreading mappings (i.e., $\| S x-$ $\left.S y\left\|^{2} \leq\right\| x-y \|^{2}+2\langle x-S x, y-S y\rangle, \forall x, y \in H\right)$. ing:

Relevant properties of $L$-hybrid mappings are the follow-

$\left(\mathscr{H}_{L}-1\right)$ If $S \in \mathscr{H}_{L}$ and $\operatorname{Fix}(S) \neq \emptyset$, then $S$ is quasinonexpansive (i.e., $\|T x-p\| \leq\|x-p\|$ for all $p \in \operatorname{Fix}(T)$, for all $x \in H$ ) (the proof is immediate by the definition).

$\left(\mathscr{H}_{L}-2\right)$ If $S \in \mathscr{H}_{L} \Rightarrow I-S$ is demiclosed in $0 \in H$ (for the proof see [1]).

$\left(\mathscr{H}_{L}-3\right)$ If $S \in \mathscr{H}_{L} \Rightarrow \operatorname{Fix}(S)$ is a closed and convex set (for the proof, see [4]).

$\left(\mathscr{H}_{L}-4\right)$ If $S \in \mathscr{H}_{L} \Rightarrow S_{\delta} \in \mathscr{H}_{L / \delta}$, where $S_{\delta}$ is the type average mapping

$$
S_{\delta}=(1-\delta) I+\delta S
$$

(for the proof, see [5]).
A first improvement of Theorem 1 [2] that one can obtain is to replace $S$ with an $L$-hybrid mapping. (This will be a corollary of our main result, thanks to $\left(\mathscr{H}_{L}-1\right),\left(\mathscr{H}_{L}-3\right)$, and next Proposition 2.)

But we can do better. Inspired by the paper of Wongchan and Saejung [6], we can consider a mapping $S$ that has only the properties of being strongly quasinonexpansive and demiclosed.

We recall that the concept of strongly nonexpansive mapping was introduced by Bruck and Reich in 1977 [7] as follows: a mapping $T$ is said to be strongly nonexpansive if $T$ is nonexpansive and whenever $\left(x_{n}-y_{n}\right)$ is bounded and $\left\|x_{n}-y_{n}\right\|-\left\|T x_{n}-T y\right\| \rightarrow 0$, it follows that $\left(x_{n}-y_{n}\right)-\left(T x_{n}-\right.$ $\left.T y_{n}\right) \rightarrow 0$.

To our knowledge, Saejung [8] in 2010 introduced the concept of strong quasi-nonexpansivity: a mapping $S$ is said strongly quasi-nonexpansive if $\operatorname{Fix}(S) \neq \emptyset, S$ is quasinonexpansive and $x_{n}-S x_{n} \rightarrow 0$ whenever $\left(x_{n}\right)$ is a bounded sequence such that $\left\|x_{n}-p\right\|-\left\|S x_{n}-p\right\| \rightarrow 0$ for some $p \in \operatorname{Fix}(S)$.

In [7] was proved that an average mapping defined on a uniformly convex Banach space is strongly nonexpansive.

Following the same line on the proof, one can show that a type average mapping $S_{\delta}=(1-\delta) I+\delta S$ of a quasinonexpansive mapping is strongly quasinonexpansive. For the sake of completeness, we propose here the following proof, lightly different, valid in Hilbert spaces.

Proposition 2. Let $S: C \rightarrow H$ be a quasinonexpansive mapping, where $C$ is a closed convex subset of $H$. Then the type average mapping $S_{\delta}=(1-\delta) I+\delta S$ is a strongly quasinonexpansive mapping.

Proof. Suppose $\left(x_{n}\right) \subset C$ is a bounded sequence such that $\left\|x_{n}-p\right\|-\left\|S_{\delta} x_{n}-p\right\| \rightarrow 0$ for some $p \in \operatorname{Fix}(S)$. Now

$$
\begin{aligned}
\left\|S_{\delta} x_{n}-p\right\| & =\left\|(1-\delta) x_{n}+\delta S x_{n}-p\right\| \\
& \leq(1-\delta)\left\|x_{n}-p\right\|+\delta\left\|S x_{n}-p\right\| \\
& \Longrightarrow\left\|x_{n}-p\right\|-\left\|S_{\delta} x_{n}-p\right\| \\
& \geq \delta\left(\left\|x_{n}-p\right\|-\left\|S x_{n}-p\right\|\right) .
\end{aligned}
$$

So

(by quasinonexpansivity of $S$ )

$$
\begin{aligned}
0 & \leq\left\|x_{n}-p\right\|-\left\|S x_{n}-p\right\| \\
& \leq \frac{1}{\delta}\left[\left\|x_{n}-p\right\|-\left\|S_{\delta} x_{n}-p\right\|\right] \longrightarrow 0 \\
\Longrightarrow & \left\|x_{n}-p\right\|-\left\|S x_{n}-p\right\| \longrightarrow 0 \text { too. }
\end{aligned}
$$

Now, let $\left(x_{n_{k}}-p\right)$ be a subsequence of $\left(x_{n}-p\right)$ that admits limit, $\left\|x_{n_{k}}-p\right\| \rightarrow d$, as $k \rightarrow \infty$. Then, from the assumption that $\left\|x_{n}-p\right\|-\left\|S_{\delta} x_{n}-p\right\| \rightarrow 0$, it follows that $\left\|S_{\delta} x_{n_{k}}-p\right\| \rightarrow d$ 
as $k \rightarrow \infty$; that is, $\left\|(1-\delta)\left(x_{n}-p\right)+\delta\left(S x_{n}-p\right)\right\| \rightarrow d$. But then

$$
\begin{aligned}
d^{2} & =\lim _{k}\left\|(1-\delta)\left(x_{n_{k}}-p\right)+\delta\left(S x_{n_{k}}-p\right)\right\|^{2} \\
& =\lim _{k}\left[(1-\delta)\left\|x_{n_{k}}-p\right\|^{2}+\delta\left\|S x_{n_{k}}-p\right\|^{2}\right. \\
& \left.-\delta(1-\delta)\left\|S x_{n_{k}}-x_{n_{k}}\right\|^{2}\right] \\
\Longrightarrow & \lim _{k}\left\|x_{n_{k}}-S x_{n_{k}}\right\|=0 \\
\Longrightarrow & \lim _{k}\left\|x_{n_{k}}-S x_{n_{k}}\right\|=\delta \lim _{k}\left\|x_{n_{k}}-S x_{n_{k}}\right\|=0 .
\end{aligned}
$$

By the boundness of the sequence $\left(x_{n}\right)$, this is sufficient, by the standard argument of compactness, to ensure $x_{n}-S_{\delta} x_{n} \rightarrow 0$ as $n \rightarrow \infty$.

So, by the quasinonexpansivity of $L$-hybrid mappings, it follows that in Theorem 1 [2] the assumption that $S$ is a strongly quasinonexpansive mapping instead of type average nonspreading (or also $L$-hybrid) mapping is a better assumption.

Of course, one can ask if some important $L$-hybrid mapping, as a nonspreading mapping, or a nonexpansive mapping, is already strongly quasinonexpansive. This is not always true, as shown in the following proposition.

Proposition 3. There exist nonexpansive mappings that are not strongly quasinonexpansive. Moreover, there exist nonspreading mappings that are not strongly quasinonexpansive.

Proof. Let $T: H \rightarrow H$ be such that $T x=-x$. Then $T$ is nonexpansive but not strongly quasinonexpansive.

Moreover let $X=A \cup B \cup C \subset H$, where

$$
\begin{aligned}
& A=\{x \in H:\|x\| \leq 1\}, \\
& B=\{x \in H: 1<\|x\|<2\}, \\
& C=\{x \in H: 2 \leq\|x\| \leq 3\} .
\end{aligned}
$$

Define $S: X \rightarrow X$ by

$$
S x:= \begin{cases}x, & \text { if } x \in A, \\ \frac{x}{\|x\|}, & \text { if } x \in B, \\ 0, & \text { if } x \in C .\end{cases}
$$

One can see that $S$ is a nonspreading mapping, distinguishing three cases: $(x \in A, y \in B),(x \in A, y \in C)$, and $(x \in B$, $y \in C)$.

To see that $S$ is not strongly quasinonexpansive, take $x_{0}$ with $\left\|x_{0}\right\|=1$.

Then $x_{0} \in \operatorname{Fix}(S)$. Moreover, define $z_{n}=(2+(1 / n)) x_{0}$. Then $S z_{n}=0$ and $\left\|z_{n}-x_{0}\right\|-\left\|S z_{n}-x_{0}\right\|=1+(1 / n)-1 \rightarrow 0$ but $S z_{n}-z_{n}=(2+(1 / n)) x_{0} \rightarrow 2 x_{0}$.

Conversely, we have the following.
Proposition 4. There exist strongly quasinonexpansive mappings that are not $L$-hybrid mappings for any $L$ (and so, thanks to $\left(\mathscr{H}_{L}-4\right)$, that are not ever type average $S_{\delta}$ with $S L$-hybrid).

Proof. Let $H=\mathbb{R}$. Define

$$
\begin{aligned}
T(0) & =0, \\
T(n) & =-n+\frac{1}{n+1}, \quad \forall n \in \mathbb{N}, n>0, \\
T(-n) & =n-\frac{1}{n+1}, \quad \forall n \in \mathbb{N}, n>0 .
\end{aligned}
$$

Then define in linear way $T$ on each interval $[n, n+1], n \in \mathbb{Z}$.

One can see easily that $\operatorname{Fix}(T)=0$ and $T$ is strongly quasinonexpansive.

Moreover, from the fact that, for large $n \in \mathbb{Z}, T$ is defined almost as $-I$, one can prove that can not be $L$-hybrid for any $L \geq 0$.

Finally, we show that the hypothesis on demiclosedness of $I-T$ is necessary in Theorem 2.3 (the main Theorem) of [6].

Proposition 5. There exist strongly quasinonexpansive mappings such that $(I-S)$ are not demiclosed in 0.

Proof. Let $H=\mathbb{R}$. Define $S:[0,1] \rightarrow[0,1]$ by

$$
S x:= \begin{cases}x^{2}, & \text { if } 0 \leq x<1, \\ \frac{1}{2}, & \text { if } x=1 .\end{cases}
$$

Then $\operatorname{Fix}(S)=\{0\}, S$ is strongly quasinonexpansive, but $I-S$ is not demiclosed on $0\left(x_{n}=1-(1 / n)\right.$ is such that $x_{n} \rightarrow 1$ but $x_{n}-S x_{n} \rightarrow 0$ ).

Reassuming, the main result of [6] has allowed us to improve Theorem 1 [2] with an emphasis on the concept of strong quasinonexpansivity instead of nonspreading.

Our reasoning is inspired by the ideas contained in $[2,6$, 9].

The solid bases on which our proof rests are given by the following lemmas.

Lemma 6 (Xu's Lemma [10]). Assume $\left(a_{n}\right)_{n \in \mathbb{N}}$ is a sequence of nonnegative numbers such that

$$
a_{n+1} \leq\left(1-s_{n}\right) a_{n}+s_{n} \sigma_{n}+\gamma_{n}, \quad n \geq 0,
$$

where $\left(s_{n}\right)_{n}$ is a sequence in $[0,1]$ and $\left(\delta_{n}\right)_{n}$ is a sequence in $\mathbb{R}$ such that
(1) $\sum_{n=1}^{\infty} s_{n}=\infty$;
(2) $\lim \sup _{n \rightarrow \infty} \sigma_{n} \leq 0$;
(3) $\gamma_{n} \geq 0, \sum_{n=1}^{\infty} \gamma_{n}<\infty$.

Then $\lim _{n \rightarrow \infty} a_{n}=0$. 
Lemma 7 (Maingé's Lemma [11]). Let $\left(\gamma_{n}\right)_{n}$ be real sequence that has a subsequence $\left(\gamma_{n_{j}}\right)$ which satisfies $\gamma_{n_{j}}<\gamma_{n_{j}+1}$ for all $j$. Then there exists an increasing sequence of integers $(\tau(n))_{n \geq n_{0}}$ satisfying

(1) $\lim _{n} \tau_{n}=+\infty$;

(2) $\gamma_{\tau(n)}<\gamma_{\tau(n)+1}$, for all $n \geq n_{0}$;

(3) $\gamma_{n}<\gamma_{\tau(n)+1}$, for all $n \geq n_{0}$.

\section{Main Results}

In all this section we denote by $O(1)$ any bounded real sequence (so, e.g., $O(1)+O(1)=O(1)$ ).

Theorem 8. Let $H$ be a Hilbert space. Suppose

(i) $T: H \rightarrow H$ is a nonexpansive mapping and $S: H \rightarrow$ $H$ is a strongly quasinonexpansive mapping such that $I-S$ is demiclosed in 0 , for which $\operatorname{Fix}(T) \cap \operatorname{Fix}(S) \neq \emptyset$;

(ii) $A: H \rightarrow H$ is a linear bounded self-adjoint $\bar{\gamma}-$ strongly positive operator, $\bar{\gamma}<1$;

(iii) $f: H \rightarrow H$ is a contraction, with $\rho<\bar{\gamma}$ (of course this is not a real restriction, but this is useful for convenience of notations);

(iv) $\left(\alpha_{n}\right)_{n},\left(\beta_{n}\right)_{n}$ are two real sequences in $[0,1]$.

Consider the iteration scheme

$$
\begin{aligned}
& x_{1} \in C \text {, } \\
& x_{n+1}=\alpha_{n} f\left(x_{n}\right)+\left(I-\alpha_{n} A\right)\left[\beta_{n} T x_{n}+\left(1-\beta_{n}\right) S x_{n}\right] \text {, } \\
& n \geq 1 \text {. }
\end{aligned}
$$

Then

(1) if $\sum_{n}\left(1-\beta_{n}\right)<\infty, \alpha_{n} \rightarrow 0, \sum_{n} \alpha_{n}=\infty$, and $\sum_{n}\left|\alpha_{n}-\alpha_{n+1}\right|<\infty$, then $\left(x_{n}\right)$ strongly converges to $\bar{p} \in \operatorname{Fix}(T)$ which is the unique point in $\operatorname{Fix}(T)$ that solves the variational inequality

$$
\langle(A-f) \bar{p}, x-\bar{p}\rangle \geq 0, \quad \forall x \in \operatorname{Fix}(T),
$$

which is the optimality condition for the minimization problem

$$
\min _{x \in \operatorname{Fix}(T)} \frac{1}{2}\langle A x, x\rangle-h(x),
$$

where $h$ is a potential function for $f\left(\right.$ i.e., $h^{\prime}(x)=f(x)$, for all $x \in H)$;

(2) if $\sum_{n} \beta_{n}<\infty, \alpha_{n} \rightarrow 0, \sum_{n} \alpha_{n}=+\infty$, and $\beta_{n} / \alpha_{n} \rightarrow$ 0 , then $\left(x_{n}\right)_{n}$ converges strongly to $\widetilde{p} \in \operatorname{Fix}(S)$ which is the unique solution in $\operatorname{Fix}(S)$ of the variational inequality

$$
\langle(A-f) \tilde{p}, x-\tilde{p}\rangle \geq 0, \quad \forall x \in \operatorname{Fix}(S) ;
$$

(3) if $\lim \inf _{n} \beta_{n}\left(1-\beta_{n}\right)>0, \alpha_{n} \rightarrow 0$, and $\sum_{n} \alpha_{n}=+\infty$, then $\left(x_{n}\right)$ strongly converges to $p_{0} \in \operatorname{Fix}(T) \cap \operatorname{Fix}(S)$ which is the unique solution in $\operatorname{Fix}(T) \cap \operatorname{Fix}(S)$ of the variational inequality

$$
\left\langle(A-f) p_{0}, x-p_{0}\right\rangle \geq 0, \quad \forall x \in \operatorname{Fix}(T) \cap \operatorname{Fix}(S) .
$$

Proof. First of all, we see that $\left(x_{n}\right)$ is a bounded sequence. Indeed, let $U_{n}=\beta_{n} T+\left(1-\beta_{n}\right) S$ and $z \in \operatorname{Fix}(T) \cap \operatorname{Fix}(S)$. Then $\left\|x_{n+1}-z\right\|=\left\|\alpha_{n} f\left(x_{n}\right)+\left(I-\alpha_{n} A\right) U_{n} x_{n}-p\right\|$ (by linearity of $A)=\|\left(I-\alpha_{n} A\right)\left(U_{n} x_{n}-z\right)+\alpha_{n}(f(z)-A z)-\alpha_{n}(f(z)-$ $\left.f\left(x_{n}\right)\right) \| \leq\left(\right.$ since $\left.\left\|I-\alpha_{n} A\right\| \leq 1-\alpha_{n} \bar{\gamma},[9]\right) \leq\left(1-\alpha_{n} \bar{\gamma}+\alpha_{n} \rho\right) \| x_{n}-$ $z\left\|+\alpha_{n}\right\| f(z)-A z\left\|=\left[1-\alpha_{n}(\bar{\gamma}-\rho)\right]\right\| x_{n}-z \|+\alpha_{n}(\bar{\gamma}-\rho)(\| f(z)-$ $A z \| / \bar{\gamma} \rho$ ) (from convexity) $\leq \max \left\{\left\|x_{n}-z\right\|,\|f(z)-A z\| / \bar{\gamma} \rho\right\}$ (from induction) $\leq \max \left\{\left\|x_{1}-z\right\|,\|f(z)-A(z)\| / \bar{\gamma} \rho\right\}$.

Then $\left(x_{n}\right)$ is bounded.

Moreover

$$
x_{n+1}-U_{n} x_{n}=\alpha_{n}\left(f\left(x_{n}\right)-A U_{n} x_{n}\right) \longrightarrow 0,
$$

$$
n \longrightarrow \infty \text {, }
$$

since $\alpha_{n} \rightarrow 0$.

Proof of (1). The the key is to prove that

$$
x_{n+1}-x_{n} \longrightarrow 0 .
$$

To see this, we calculate

$$
\begin{aligned}
& \left\|x_{n+1}-x_{n}\right\|=\|\left(I-\alpha_{n} A\right)\left(U_{n} x_{n}-U_{n-1} x_{n-1}\right)-\left(\alpha_{n}\right. \\
& \left.-\alpha_{n-1}\right) A U_{n-1} x_{n-1}+\alpha_{n}\left(f\left(x_{n}\right)-f\left(x_{n-1}\right)\right)+\left(\alpha_{n}\right. \\
& \left.-\alpha_{n-1}\right) f\left(x_{n-1}\right)\|=\|\left(I-\alpha_{n} A\right)\left(U_{n} x_{n}-U_{n-1} x_{n-1}\right) \\
& +\left(\alpha_{n-1}-\alpha_{n}\right)\left[A U_{n-1} x_{n-1}-f\left(x_{n-1}\right)\right] \\
& +\alpha_{n}\left(f\left(x_{n}\right)-f\left(x_{n-1}\right)\right)\|=\|\left(\alpha_{n-1}-\alpha_{n}\right) \\
& \quad \cdot\left(A U_{n-1} x_{n-1}-f\left(x_{n-1}\right)\right)+\alpha_{n}\left(f\left(x_{n}\right)\right. \\
& \left.-f\left(x_{n-1}\right)\right)+\left(I-\alpha_{n} A\right)\left[\beta_{n} T x_{n}+\left(1-\beta_{n}\right) S x_{n}\right. \\
& \left.-\beta_{n-1} T x_{n-1}-\left(1-\beta_{n-1}\right) S x_{n-1}\right]\|=\|\left(\alpha_{n-1}-\alpha_{n}\right) \\
& \quad \cdot\left(A U_{n-1} x_{n-1}-f\left(x_{n-1}\right)\right)+\alpha_{n}\left(f\left(x_{n}\right)\right. \\
& \left.-f\left(x_{n-1}\right)\right)+\left(I-\alpha_{n} A\right)\left[\beta_{n}\left(T x_{n}-T x_{n-1}\right)\right. \\
& \quad+\left(1-\beta_{n}\right)\left(S x_{n}-S x_{n-1}\right)+\left(\beta_{n}-\beta_{n-1}\right) T x_{n-1} \\
& \left.\quad+\left(\beta_{n-1}-\beta_{n}\right) S x_{n-1}\right] \| \\
& \quad \Longrightarrow(\text { by nonexpansivity of } T), \\
& \left\|x_{n+1}-x_{n}\right\| \leq\left|\alpha_{n-1}-\alpha_{n}\right| O(1)+\alpha_{n} \rho\left\|x_{n}-x_{n-1}\right\| \\
& \quad+\left(1-\alpha_{n} \bar{\gamma}\right)\left[\beta_{n}\left\|x_{n}-x_{n-1}\right\|+\left(1-\beta_{n}\right) O(1)+\mid \beta_{n}\right. \\
& \left.-\beta_{n-1} \mid O(1)\right]=\left(1-s_{n}\right)\left\|x_{n}-x_{n-1}\right\|+\gamma_{n},
\end{aligned}
$$

where $s_{n}=1-\beta_{n}+\alpha_{n}\left(\bar{\gamma} \beta_{n}-\rho\right) \geq \alpha_{n} O(1)$; definitively, $\gamma_{n}=$ $\left|\alpha_{n-1}-\alpha_{n}\right| O(1)+\left[\left(1-\alpha_{n} \bar{\gamma}\right)\left(1-\beta_{n}\right)+\left|\beta_{n}-\beta_{n-1}\right|\right] O(1)$. 
Thanks to hypotheses on $\alpha_{n}, \beta_{n}$, we see that $s_{n} \rightarrow 0$, $\sum_{n} s_{n}=+\infty$, and $\sum_{n} \gamma_{n}<\infty$. This is sufficient, from Xu's Lemma, to conclude $x_{n+1}-x_{n} \rightarrow 0$.

From this and (22), it follows immediately that

$$
x_{n}-U_{n} x_{n} \longrightarrow 0 .
$$

Indeed, $x_{n}-U_{n} x_{n}=\left(x_{n}-x_{n+1}\right)-\left(x_{n+1}-U_{n} x_{n}\right)$.

Moreover

$$
\begin{aligned}
\left\|x_{n}-U_{n} x_{n}\right\| & =\left\|x_{n}-\beta_{n} T x_{n}-\left(1-\beta_{n}\right) S x_{n}\right\| \\
& \geq\left\|x_{n}-\beta_{n} T x_{n}\right\|-\left(1-\beta_{n}\right)\left\|S x_{n}\right\| \\
& \Longrightarrow\left\|x_{n}-\beta_{n} T x_{n}\right\| \\
& \leq\left\|x_{n}-U_{n} x_{n}\right\|+\left(1-\beta_{n}\right) O(1),
\end{aligned}
$$

and so from (25) and hypotheses $\sum_{n}\left(1-\beta_{n}\right)<\infty$, we have also

$$
x_{n}-\beta_{n} T x_{n} \longrightarrow 0 .
$$

From this we deduce also that

$$
x_{n}-T x_{n} \longrightarrow 0,
$$

and this gives that any weak limit of $\left(x_{n}\right)$ is in $\operatorname{Fix}(T)$, since $T$ is nonexpansive, and thus the Principle of Demiclosedness is satisfied.

Now we can show that $x_{n} \rightarrow \bar{p}$, where $\bar{p}$ is the unique solution in $\operatorname{Fix}(T)$ of variational inequality (18). We show first that

$$
\limsup _{n}\left\langle U_{n} x_{n}-\bar{p}, f(\bar{p})-A \bar{p}\right\rangle \leq 0 .
$$

Indeed, let $\left(x_{n_{k}}\right)$ be a subsequence of $\left(x_{n}\right)$ such that

$$
\begin{aligned}
& \limsup _{n}\left\langle x_{n}-\bar{p}, f(\bar{p})-A \bar{p}\right\rangle \\
& \quad=\lim _{k}\left\langle x_{n_{k}}-\bar{p}, f(\bar{p})-A \bar{p}\right\rangle,
\end{aligned}
$$

and $x_{n_{k}} \rightarrow z$. Then $z \in \operatorname{Fix}(T)$ and so, from (30),

$$
\limsup _{n}\left\langle x_{n}-\bar{p}, f(\bar{p})-A \bar{p}\right\rangle=\langle z-\bar{p}, f(\bar{p})-A \bar{p}\rangle,
$$

and this is nonpositive by definition of $\bar{p}$.

So,

$$
\begin{aligned}
& \limsup _{n}\left\langle U_{n} x_{n}-\bar{p}, f(\bar{p})-A \bar{p}\right\rangle \\
& \quad=\limsup _{n}\left[\left\langle x_{n}-\bar{p}, f(\bar{p})-A \bar{p}\right\rangle\right. \\
& \left.\quad+\left\langle U_{n} x_{n}-x_{n}, f(\bar{p})-A \bar{p}\right\rangle\right] \quad(\text { from (25)) } \\
& =\limsup _{n}\left\langle x_{n}-\bar{p}, f(\bar{p})-A \bar{p}\right\rangle \quad(\text { by (30)) } \\
& \leq 0 .
\end{aligned}
$$

Finally

$$
\begin{aligned}
\| x_{n+1} & -\bar{p} \| \\
= & \left\|\left(I-\alpha_{n} A\right)\left(U_{n} x_{n}-\bar{p}\right)+\alpha_{n}\left(f\left(x_{n}\right)-A \bar{p}\right)\right\|^{2} \\
\leq & \left(1-\alpha_{n} \bar{\gamma}\right)^{2}\left\|U_{n} x_{n}-\bar{p}\right\|^{2}+\alpha_{n}^{2}\left\|f\left(x_{n}\right)-A \bar{p}\right\|^{2} \\
& +2 \alpha_{n}\left\langle\left(I-\alpha_{n} A\right)\left(U_{n} x_{n}-\bar{p}\right), f\left(x_{n}\right)-A \bar{p}\right\rangle \\
\leq & \left(1-\alpha_{n} \bar{\gamma}\right)^{2}\left\|\beta_{n}\left(T x_{n}-\bar{p}\right)+\left(1-\beta_{n}\right)\left(S x_{n}-\bar{p}\right)\right\|^{2} \\
& +\alpha_{n}^{2}\left\|f\left(x_{n}\right)-A \bar{p}\right\|^{2} \\
& +2 \alpha_{n}\left\langle U_{n} x_{n}-\bar{p}, f\left(x_{n}\right)-A \bar{p}\right\rangle \\
& -2 \alpha_{n}^{2}\left\langle A\left(U_{n} x_{n}-\bar{p}\right), f\left(x_{n}\right)-A \bar{p}\right\rangle \\
\leq & \left(1-\alpha_{n} \bar{\gamma}\right)^{2}\left[\beta_{n}\left\|x_{n}-\bar{p}\right\|+\left(1-\beta_{n}\right) O(1)\right]^{2} \\
& +\alpha_{n}^{2} O(1)+2 \alpha_{n}\left\langle U_{n} x_{n}-\bar{p}, f\left(x_{n}\right)-A \bar{p}\right\rangle \\
& +2 \alpha_{n}^{2} O(1) \\
\leq & \left(1-\alpha_{n} \bar{\gamma}\right)^{2}\left\|x_{n}-\bar{p}\right\|^{2}+\left(1-\beta_{n}\right) O(1)+\alpha_{n}^{2} O(1) \\
& +2 \alpha_{n}\left\langle U_{n} x_{n}-\bar{p}, f\left(x_{n}\right)-f(\bar{p})\right\rangle \\
& +2 \alpha_{n}\left\langle U_{n} x_{n}-\bar{p}, f(\bar{p})-A \bar{p}\right\rangle \\
\leq & \left(1-\alpha_{n} \bar{\gamma}\right)^{2}\left\|x_{n}-\bar{p}\right\|^{2}+\left(1-\beta_{n}\right) O(1)+\alpha_{n}^{2} O(1) \\
& +2 \alpha_{n}\left\langle U_{n} x_{n}-\bar{p}+f(\bar{p})-A \bar{p}\right\rangle \\
& +2 \alpha_{n}\left[\beta_{n}\left\|x_{n}-\bar{p}\right\|+\left(1-\beta_{n}\right) O(1)\right] \rho\left\|x_{n}-\bar{p}\right\| \\
\leq & {\left[\left(1-\alpha_{n} \bar{\gamma}\right)^{2}+2 \alpha_{n} \rho\right]\left\|x_{n}-\bar{p}\right\|^{2} } \\
& +\alpha_{n}\left[\alpha_{n} O(1)+2\left\langle U_{n} x_{n}-\bar{p}, f(\bar{p})-A \bar{p}\right\rangle\right] \\
& +\left(1-\beta_{n}\right) O(1) \\
= & \left(1-s_{n}\right)\left\|x_{n}-\bar{p}\right\|^{2}+\alpha_{n} \sigma_{n}+\gamma_{n}, \\
&
\end{aligned}
$$

where

$$
\begin{aligned}
s_{n} & =1-\left(1-\alpha_{n} \bar{\gamma}\right)^{2}-2 \alpha_{n} \rho \\
& =1-1-\alpha_{n}^{2} \bar{\gamma}^{2}+2 \alpha_{n} \bar{\gamma}-2 \alpha_{n} \rho \\
& =\alpha_{n}\left[\alpha_{n} \bar{\gamma}^{2}+2(\bar{\gamma}-\rho)\right], \\
\sigma_{n} & =\alpha_{n} O(1)+2\left\langle U_{n} x_{n}-\bar{p}, f(\bar{p})-A \bar{p}\right\rangle, \\
\gamma_{n} & =\left(1-\beta_{n}\right) O(1) .
\end{aligned}
$$

Thanks to the hypotheses on $\alpha_{n}, \beta_{n}$ and (29), from Xu's Lemma again, we obtain $x_{n} \rightarrow \bar{p}$.

Proof of (2). Let $\widetilde{p}$ be the unique solution of variational inequality (20). 
We want to show that $x_{n} \rightarrow \widetilde{p}$. Now,

$$
\begin{aligned}
& \left\|x_{n+1}-\tilde{p}\right\|^{2} \\
& =\left\|\alpha_{n} f\left(x_{n}\right)+\left(I-\alpha_{n} A\right) U_{n} x_{n}-\widetilde{p}+\alpha_{n} \tilde{p}-\alpha_{n} \widetilde{p}+\alpha_{n} A z-\alpha_{n} A z\right\|^{2} \\
& =\left\|\alpha_{n}\left(f\left(x_{n}\right)-A z\right)+\left(I-\alpha_{n} A\right)\left(U_{n} x_{n}-\widetilde{p}\right)\right\|^{2}
\end{aligned}
$$$$
\text { (by the subdifferential inequality }\|x+y\|^{2} \leq\|x\|^{2}+2\langle y, x+y\rangle \text { ) }
$$

$$
\begin{aligned}
\leq & \left(1-\alpha_{n} \bar{\gamma}\right)^{2}\left\|U_{n} x_{n}-\tilde{p}\right\|^{2}+2 \alpha_{n}\left\langle f\left(x_{n}\right)-A \tilde{p}, x_{n+1}-\tilde{p}\right\rangle \\
= & \left(1-\alpha_{n} \bar{\gamma}\right)^{2}\left\|\beta_{n}\left(T x_{n}-\tilde{p}\right)+\left(1-\beta_{n}\right)\left(S x_{n}-\tilde{p}\right)\right\|^{2} \\
& +2 \alpha_{n}\left\langle f\left(x_{n}\right)-f(\tilde{p}), x_{n+1}-\tilde{p}\right\rangle \\
& +2 \alpha_{n}\left\langle f(\tilde{p})-A \tilde{p}, x_{n+1}-\tilde{p}\right\rangle
\end{aligned}
$$

(by the quasinonexpansivity of $S$ )

$$
\begin{aligned}
& \leq\left(1-\alpha_{n} \bar{\gamma}\right)^{2}\left\|x_{n}-\tilde{p}\right\|^{2}+\beta_{n} O(1)+2 \alpha_{n} \rho\left\|x_{n}-\tilde{p}\right\|\left\|x_{n+1}-\tilde{p}\right\| \\
& \quad+2 \alpha_{n}\left\langle x_{n+1}-\tilde{p}, f(\tilde{p})-A \tilde{p}\right\rangle .
\end{aligned}
$$

At this point we distinguish two cases: or the sequence $\| x_{n}-$ $\widetilde{p} \|$ is definitively not increasing or not.

Alternative 1. $\left(\left\|x_{n}-\widetilde{p}\right\|\right)$ is definitively not increasing, so $\left\|x_{n+1}-\widetilde{p}\right\| \leq\left\|x_{n}-\tilde{p}\right\|, \forall n \geq N$.

Then (35) furnish

$$
\begin{aligned}
\left\|x_{n+1}-\tilde{p}\right\|^{2} \leq & {\left[\left(1-\alpha_{n} \bar{\gamma}\right)^{2}+2 \alpha_{n} \rho\right]\left\|x_{n}-\tilde{p}\right\| } \\
& +\beta_{n} O(1) \\
& +2 \alpha_{n}\left\langle x_{n+1}-\tilde{p}-f(\widetilde{p})-A \widetilde{p}\right\rangle .
\end{aligned}
$$

Putting $s_{n}=1-\left(1-\alpha_{n} \bar{\gamma}\right)^{2}-2 \alpha_{n} \rho=\alpha_{n} \bar{\gamma}^{2}-2 \alpha_{n} \bar{\gamma}+2 \alpha_{n} \rho=$ $\alpha_{n}\left[\alpha_{n} \bar{\gamma}^{2}+2(\bar{\gamma}-\rho)\right] \geq 0, \sigma_{n}=\left\langle x_{n+1}-\tilde{p}, f(\tilde{p})-A \tilde{p}\right\rangle$, and $\gamma_{n}=\beta_{n} O(1)$, we can rewrite (36) as

$$
\left\|x_{n+1}-\tilde{p}\right\|^{2} \leq\left(1-s_{n}\right)\left\|x_{n}-\tilde{p}\right\|^{2}+\alpha_{n} \sigma_{n}+\gamma_{n}
$$

so the thesis $x_{n} \rightarrow \widetilde{p}$ will follow again by Xu's Lemma if we are able to show that

$$
\limsup _{n}\left\langle x_{n+1}-\tilde{p}, f(\widetilde{p})-A \widetilde{p}\right\rangle \leq 0
$$

(Note that until now we have not used the hypothesis of strong quasinonexpansivity of $S$.) Now, since $\left(\left\|x_{n}-\tilde{p}\right\|\right)$ is definitively not increasing, there exists the $\lim _{n}\|x-n-\tilde{p}\|$. Then

$$
\begin{aligned}
0= & \lim _{n}\left\|x_{n+1}-\tilde{p}\right\|-\left\|x_{n}-\tilde{p}\right\| \\
& \leq \lim _{n} \inf \left[\alpha_{n}\left\|f\left(x_{n}\right)-A \widetilde{p}\right\|\right. \\
& \left.+\left(1-\alpha_{n} \bar{\gamma}\right)\left\|U_{n} x_{n}-\tilde{p}\right\|-\left\|x_{n}-\tilde{p}\right\|\right]
\end{aligned}
$$

(since $\left.\alpha_{n} \longrightarrow 0\right)$

$$
\begin{aligned}
& =\lim _{n} \inf \left[\left\|U_{n} x_{n}-\tilde{p}\right\|-\left\|x_{n}-\tilde{p}\right\|\right] \\
& \quad=\lim _{n} \inf \left\|\beta_{n}\left(T x_{n}-\tilde{p}\right)+\left(1-\beta_{n}\right)\left(S x_{n}-\tilde{p}\right)\right\| \\
& \quad-\left\|x_{n}-\tilde{p}\right\|\left(\text { by hypothesis } \beta_{n} \longrightarrow 0\right) \\
& \quad=\lim _{n} \inf \left\|S x_{n}-\tilde{p}\right\|-\left\|x_{n}-\tilde{p}\right\| \leq \liminf _{n}\left\|S x_{n}-\tilde{p}\right\| \\
& \quad-\left\|x_{n}-\tilde{p}\right\| \quad(\text { by quasinonexpansivity of } S) \\
& \leq \limsup _{n}\left\|x_{n}-\tilde{p}\right\|-\left\|x_{n}-\tilde{p}\right\|=0 .
\end{aligned}
$$

Thus

$$
\lim _{n}\left\|S x_{n}-\tilde{p}\right\|-\left\|x_{n}-\tilde{p}\right\|=0 .
$$

From the strong quasinonexpansivity of $S$, we deduce

$$
S x_{n}-x_{n} \longrightarrow 0 \text {. }
$$

At this point, by using the demiclosedness of $(I-S)$ in 0 , we can proceed as in the Proof of (1) to show (38).

The statement is proved when Alternative 1 holds.

Alternative 2. $\left(\left\|x_{n}-\widetilde{p}\right\|\right)$ is not definitively not increasing; that is, there exists a subsequence $\left(\left\|x_{n_{j}}-\widetilde{p}\right\|\right)$ such that

$$
\left\|x_{n_{j}}-\tilde{p}\right\|<\left\|x_{n_{j}+1}-\tilde{p}\right\|, \quad \forall j \in \mathbb{N} .
$$

From Maingé's Lemma, it follows that there exists an increasing sequence of integers $(\tau(n))_{n \in \ltimes}$ satisfying

$$
\begin{aligned}
\lim _{n} \tau(n) & =+\infty, \\
\left\|x_{\tau(n)}-\tilde{p}\right\| & <\left\|x_{\tau(n)+1}-\tilde{p}\right\|, \\
\left\|x_{n}-\tilde{p}\right\| & <\left\|x_{\tau(n)}-\tilde{p}\right\|, \quad \forall n \geq n_{0} .
\end{aligned}
$$

Then

$$
0 \leq \liminf _{n}\left\|x_{\tau(n)+1}-\tilde{p}\right\|-\left\|x_{\tau(n)}-\tilde{p}\right\| .
$$

Retracing the same inequalities used to have (41) with $\tau(n)$ instead of $n$, we obtain

$$
\lim _{n}\left\|S x_{\tau(n)}-\tilde{p}\right\|-\left\|x_{\tau(n)}-\tilde{p}\right\|=0 .
$$

Again the strong quasinonexpansivity yields

$$
S x_{\tau(n)}-x_{\tau(n)} \longrightarrow 0,
$$

And, from the demiclosedness of $I-S$ in 0 , we deduce as above that

$$
\limsup _{n}\left\langle x_{\tau(n)+1}-\tilde{p}, f(\widetilde{p})-A \tilde{p}\right\rangle \leq 0
$$

Incidentally, we observe that

$$
\begin{aligned}
\left\|x_{\tau(n)+1}-x_{\tau(n)}\right\|= & \alpha_{\tau(n)}\left\|\left(f\left(x_{\tau(n)}\right)-A x_{\tau(n)}\right)\right\| \\
& +\left(1-\alpha_{\tau(n)} \bar{\gamma}\right) \beta_{\tau(n)} O(1) \\
& +\left(1-\alpha_{\tau(n)} \bar{\gamma}\right)\left\|S x_{\tau(n)}-x_{\tau(n)}\right\|,
\end{aligned}
$$

and so, from (47), it follows also that $x_{\tau(n)+1}-x_{\tau(n)} \rightarrow 0$. 
Now rewrite (35) with $\tau(n)$ instead of $n$ :

$$
\begin{aligned}
& \left\|x_{\tau(n)+1}-\tilde{p}\right\| \\
& \leq\left(1-\alpha_{\tau(n)} \bar{\gamma}\right)^{2}\left\|x_{\tau(n)}-\tilde{p}\right\|^{2} \\
& \quad+2 \alpha_{\tau(n)} \rho\left\|x_{\tau(n)}-\tilde{p}\right\|\left\|x_{\tau(n)+1}-\tilde{p}\right\| \\
& \quad+2 \alpha_{\tau(n)}\left\langle x_{\tau(n)+1}-\tilde{p}, f(\tilde{p})-A \widetilde{p}\right\rangle+\beta_{\tau(n)} O(1)
\end{aligned}
$$

(by property (43))

$$
\begin{aligned}
\leq & {\left[\left(1-\alpha_{\tau(n)} \bar{\gamma}\right)^{2}+2 \alpha_{\tau(n)} \rho\right]\left\|x_{\tau(n)+1}-\tilde{p}\right\|^{2} } \\
& +2 \alpha_{\tau(n)}\left\langle x_{\tau(n)+1}-\tilde{p}, f(\widetilde{p})-A \widetilde{p}\right\rangle+\beta_{\tau(n)} O(1) \\
\Longrightarrow & 2 \alpha_{\tau(n)}(\bar{\gamma}-\rho)\left\|x_{\tau(n)+1}-\tilde{p}\right\|^{2} \\
\leq & \left(\alpha_{\tau(n)} \bar{\gamma}\right)^{2}\left\|x_{\tau(n)+1}-\tilde{p}\right\|^{2} \\
& +2 \alpha_{\tau(n)}\left\langle x_{\tau(n)+1}-\tilde{p}, f(\tilde{p})-A \tilde{p}\right\rangle+\beta_{\tau(n)} O(1) \Longrightarrow
\end{aligned}
$$

dividing by $\alpha_{\tau(n)}$

$$
\begin{aligned}
0 \leq & 2(\bar{\gamma}-\rho)\left\|x_{\tau(n)+1}-\tilde{p}\right\|^{2} \\
\leq & \alpha_{\tau(n)} \bar{\gamma}^{2}\left\|x_{\tau(n)+1}-\tilde{p}\right\|^{2} \\
& +2\left\langle x_{\tau(n)+1}-\tilde{p}, f(\tilde{p})-A \tilde{p}\right\rangle+\frac{\beta_{\tau(n)}}{\alpha_{\tau(n)}} O(1) .
\end{aligned}
$$

Passing to limsup and recalling the hypothesis $\beta_{n} / \alpha_{n} \rightarrow 0$ and (48), we obtain $\lim _{n}\left\|x_{\tau(n)}-\tilde{p}\right\| \leq \lim _{n}\left\|x_{\tau(n)+1}-\widetilde{p}\right\|=0$. Equation (44) ensures that also $x_{n} \rightarrow \tilde{p}$.

Proof of (3). Let $p_{0}$ be the unique point in $\operatorname{Fix}(S) \cap F$ that satisfies variational inequality (21). Then

$$
\begin{aligned}
\left\|U_{n} x_{n}-p_{0}\right\|^{2} & \\
= & \left\|\beta_{n}\left(T x_{n}-p_{0}\right)+\left(1-\beta_{n}\right)\left(S x_{n}-p_{0}\right)\right\|^{2} \\
= & \beta_{n}\left\|T x_{n}-p_{0}\right\|^{2}+\left(1-\beta_{n}\right)\left\|S x_{n}-p_{0}\right\|^{2} \\
& -\beta_{n}\left(1-\beta_{n}\right)\left\|T x_{n}-S x_{n}\right\|^{2} \\
\leq & \left\|x_{n}-p_{0}\right\|^{2}-\beta_{n}\left(1-\beta_{n}\right)\left\|T x_{n}-S x_{n}\right\|^{2} \Longrightarrow \\
\| x_{n+1} & -p_{0} \|^{2} \\
= & \left\|\alpha_{n}\left(f\left(x_{n}\right)-A p_{0}\right)+\left(I-\alpha_{n} A\right)\left(U_{n} x_{n}-p_{0}\right)\right\|^{2} \\
\leq & \left(1-\alpha_{n} \bar{\gamma}\right)^{2}\left\|U_{n} x_{n}-p_{0}\right\|^{2}+\alpha_{n}^{2} O(1)+\alpha_{n} O(1) \\
\leq & \left\|x_{n}-p_{0}\right\|^{2}-\beta_{n}\left(1-\beta_{n}\right)\left\|T x_{n}-S x_{n}\right\|^{2} \\
& +\alpha_{n}^{2} O(1)+\alpha_{n} O(1) .
\end{aligned}
$$

Also now we distinguish two cases.

Alternative 1. $\left(\left\|x_{n}-p_{0}\right\|\right)$ is definitively not increasing, $\| x_{n+1}-$ $p_{0}\|\leq\| x_{n}-p_{0} \|, \forall n \geq N$.
Then there exists $\lim _{n}\left\|x_{n}-\widetilde{p}\right\|$, so (52) furnish

$$
\begin{aligned}
& \beta_{n}\left(1-\beta_{n}\right)\left\|T x_{n}-S x_{n}\right\| \\
& \quad \leq\left\|x_{n}-p_{0}\right\|^{2}-\left\|x_{n+1}-p_{0}\right\|^{2}+\alpha_{n}^{2} O(1)+\alpha_{n} O(1),
\end{aligned}
$$

and so, by hypothesis $\lim _{\inf _{n}} \beta_{n}\left(1-\beta_{n}\right)>0$, we deduce

$$
T x_{n}-S x_{n} \longrightarrow 0 \text {. }
$$

Moreover,

$$
\begin{aligned}
0= & \lim _{n}\left\|x_{n+1}-p_{0}\right\|-\left\|x_{n}-p_{0}\right\| \\
& \leq \liminf \left[\alpha_{n}\left\|f\left(x_{n}\right)-A p_{0}\right\|\right. \\
& \left.+\left(1-\alpha_{n} \bar{\gamma}\right)\left\|U_{n} x_{n}-p_{0}\right\|-\left\|x_{n}-p_{0}\right\|\right] \\
\leq & \liminf _{n}\left\|U_{n} x_{n}-p_{0}\right\|-\left\|x_{n}-p_{0}\right\| \\
& =\lim _{n} \inf \left\|\beta_{n}\left(T x_{n}-p_{0}\right)+\left(1-\beta_{n}\right)\left(S x_{n}-p_{0}\right)\right\| \\
& -\left\|x_{n}-p_{0}\right\| \leq \liminf _{n} \beta_{n}\left[\left\|T x_{n}-p_{0}\right\|-\left\|x_{n}-p_{0}\right\|\right] \\
& +\left(1-\beta_{n}\right)\left[\left\|S x_{n}-p_{0}\right\|-\left\|x_{n}-p_{0}\right\|\right] \\
& \leq \limsup _{n} \beta_{n}\left[\left\|T x_{n}-p_{0}\right\|-\left\|x_{n}-p_{0}\right\|\right]+\left(1-\beta_{n}\right) \\
& +\left[\left\|S x_{n}-p_{0}\right\|-\left\|x_{n}-p_{0}\right\|\right]
\end{aligned}
$$

(by the quasinonexpansivity of $T$ and $S$ )

$\leq 0$.

But then

$$
\begin{aligned}
& \lim _{n}\left\{\beta_{n}\left[\left\|T x_{n}-p_{0}\right\|-\left\|x_{n}-p_{0}\right\|\right]\right. \\
& \left.\quad+\left(1-\beta_{n}\right)\left[\left\|S x_{n}-p_{0}\right\|-\left\|x_{n}-p_{0}\right\|\right]\right\}=0 .
\end{aligned}
$$

Since both of the addends are nonpositive and the limit of the sum is zero, it follows that

$$
\begin{aligned}
& \lim _{n} \beta_{n}\left[\left\|T x_{n}-p_{0}\right\|-\left\|x_{n}-p_{0}\right\|\right] \\
& \quad=\lim _{n}\left(1-\beta_{n}\right)\left[\left\|S x_{n}-p_{0}\right\|-\left\|x_{n}-p_{0}\right\|\right]=0 .
\end{aligned}
$$

But then the hypothesis $\lim \inf _{n} \beta_{n}\left(1-\beta_{n}\right)>0$ implies that

$$
\begin{aligned}
\lim _{n} & \left\|T x_{n}-p_{0}\right\|-\left\|x_{n}-p_{0}\right\| \\
& =\lim _{n}\left\|S x_{n}-p_{0}\right\|-\left\|x_{n}-p_{0}\right\|=0 .
\end{aligned}
$$

From strong quasinonexpansivity of $S$, it follows that

$$
S x_{n}-x_{n} \longrightarrow 0 \text {. }
$$

Again $x_{n}-T x_{n}=x_{n}-S x_{n}+S x_{n}-T x_{n}$, so, by (54) and (57),

$$
x_{n}-T x_{n} \longrightarrow 0 \text {. }
$$


We show now that

$$
\limsup _{n}\left\langle x_{n}-p_{0}, f\left(p_{0}\right)-A p_{0}\right\rangle \leq 0
$$

Indeed, select a subsequence $x_{n_{k}} \rightarrow z$ such that

$$
\begin{gathered}
\limsup _{n}\left\langle x_{n}-p_{0}, f\left(p_{0}\right)-A p_{0}\right\rangle \\
=\lim _{k}\left\langle x_{n_{k}}-p_{0}, f\left(p_{0}\right)-A p_{0}\right\rangle \\
=\left\langle z-p_{0}, f\left(p_{0}\right)-A p_{0}\right\rangle .
\end{gathered}
$$

But by the demiclosedness of both $T$ and $S$ and by (59) and (60), one deduces that

$z \in \operatorname{Fix}(S) \cap \operatorname{Fix}(T)$, and so, by definition of $p_{0}$,

(61) is obtained.

Moreover, from (59) and (60) at once, it follows that

$$
U_{n} x_{n}-x_{n} \longrightarrow 0
$$

Finally we are able to show $x_{n} \rightarrow p_{0}$. Indeed,

$$
\begin{aligned}
& \left\|x_{n+1}-p_{0}\right\|^{2}=\|\left(I-\alpha_{n} A\right)\left(U_{n} x_{n}-p_{0}\right) \\
& \quad+\alpha_{n}\left(f\left(x_{n}\right)-A p_{0}\right)\left\|^{2}=\left(1-\alpha_{n} \bar{\gamma}\right)^{2}\right\| U_{n} x_{n}-p_{0} \|^{2} \\
& \quad+\alpha_{n}^{2}\left\|f\left(x_{n}\right)-A p_{0}\right\|^{2} \\
& \quad+2 \alpha_{n}\left\langle\left(I-\alpha_{n} A\right)\left(U_{n} x_{n}-p_{0}\right), f\left(x_{n}\right)-A p_{0}\right\rangle \\
& \quad \leq\left(1-\alpha_{n} \bar{\gamma}\right)^{2}\left\|x_{n}-p_{0}\right\|^{2}+\alpha_{n}^{2}\left[\left\|f\left(x_{n}\right)-A p_{0}\right\|^{2}\right. \\
& \left.-2\left\langle A\left(U_{n} x_{n}-p_{0}\right), f\left(x_{n}\right)-A p_{0}\right\rangle\right]+2 \alpha_{n}\left\langle U_{n} x_{n}\right. \\
& \left.\quad-x_{n}, f\left(x_{n}\right)-A p_{0}\right\rangle+2 \alpha_{n}\left\langle x_{n}-p_{0}, f\left(x_{n}\right)\right. \\
& \left.\quad-f\left(p_{0}\right)\right\rangle+2 \alpha_{n}\left\langle x_{n}-p_{0}, f\left(p_{0}\right)-A p_{0}\right\rangle \\
& \quad \leq\left[\left(1-\alpha_{n} \bar{\gamma}\right)^{2}+2 \alpha_{n} \rho\right]\left\|x_{n}-p_{0}\right\|^{2}+\alpha_{n}^{2} O(1) \\
& \quad+2 \alpha_{n}\left\langle U_{n} x_{n}-x_{n}, f\left(x_{n}\right)-A p_{0}\right\rangle+2 \alpha_{n}\left\langle x_{n}\right. \\
& \left.\quad-p_{0}, f\left(p_{0}\right)-p_{0}\right\rangle .
\end{aligned}
$$

Put

$$
\begin{aligned}
s_{n}= & 1-(1-\alpha \bar{\gamma})^{2}-2 \alpha_{n} \rho, \\
\sigma_{n}= & 2\left\langle U_{n} x_{n}-x_{n}, f\left(x_{n}\right)-A p_{0}\right\rangle \\
& +2\left\langle x_{n}-p_{0}, f\left(p_{0}\right)-p_{0}\right\rangle+\alpha_{n} O(1),
\end{aligned}
$$

and the thesis follows again by Xu's Lemma, taking account of (61) and (64).

Alternative 2. $\left(\left\|x_{n}-p_{0}\right\|\right)$ is not definitively not increasing; that is, there exists a subsequence $\left(\left\|x_{n_{j}}-p_{0}\right\|\right)$ such that $\left\|x_{n_{j}}-p_{0}\right\|<$ $\left\|x_{n_{j+1}}-p_{0}\right\|, \forall j \in \mathbb{N}$.
From Maingé's Lemma, it follows that there exists an increasing sequence of integers $(\tau(n))_{n \in \mathbb{N}}$ satisfying (43) and (44).

Then

$$
\begin{aligned}
0 \leq & \liminf _{n}\left\|x_{\tau(n)+1}-p_{0}\right\|-\left\|x_{\tau(n)}-p_{0}\right\| \\
& \leq \limsup _{n}\left\|x_{\tau(n)+1}-p_{0}\right\|-\left\|x_{\tau(n)}-p_{0}\right\| \\
& \leq \underset{n}{\lim \sup _{n}}\left\|x_{n+1}-p_{0}\right\|-\left\|x_{n}-p_{0}\right\| \\
& \leq \limsup _{n} \|\left[\left(I-\alpha_{n} A\right)\left(U_{n} x_{n}-p_{0}\right)\right. \\
& \left.+\alpha_{n}\left(f\left(x_{n}\right)-A p_{0}\right)\right]\|-\| x_{n}-p_{0}\left\|\leq\left(1-\alpha_{n} \bar{\gamma}\right)\right\| x_{n} \\
& -p_{0}\left\|+\alpha_{n} O(1)-\right\| x_{n}-p_{0} \| \longrightarrow 0, \quad \text { as } n \longrightarrow \infty .
\end{aligned}
$$

Hence

$$
\lim _{n}\left[\left\|x_{\tau(n)+1}-p_{0}\right\|-\left\|x_{\tau(n)}-p_{0}\right\|\right]=0 .
$$

Now, retracing the same inequalities used to obtain (59) with $\tau(n)$ instead of $n$, we have

$$
S x_{\tau(n)}-x_{\tau(n)} \longrightarrow 0 .
$$

Moreover, we can rewrite (52) as

$$
\begin{aligned}
0 & \leq \beta_{\tau(n)}\left(1-\beta_{\tau(n)}\right)\left\|T x_{\tau(n)}-S x_{\tau(n)}\right\|^{2} \\
& \leq\left\|x_{\tau(n)}-p_{0}\right\|^{2}-\left\|x_{\tau(n)+1}-p_{0}\right\|^{2}+\alpha_{n} O(1),
\end{aligned}
$$

and so, from (68) and the hypothesis $\liminf _{n} \beta_{n}\left(1-\beta_{n}\right)>0$,

$$
T x_{\tau(n)}-S x_{\tau(n)} \longrightarrow 0 .
$$

Again,

$$
x_{\tau(n)}-T x_{\tau(n)}=x_{\tau(n)}-S x_{\tau(n)}+S x_{\tau(n)}-T x_{\tau(n)},
$$

so, by (69) and (71),

$$
x_{\tau(n)}-T x_{\tau(n)} \longrightarrow 0,
$$

so also

$$
x_{\tau(n)}-U_{\tau(n)} x_{\tau(n)} \longrightarrow 0 .
$$

The same reasoning used to have (61) can be now repeated with $\tau(n)$ instead of $n$ obtaining

$$
\limsup _{n}\left\langle x_{\tau(n)}-p_{0}, f\left(p_{0}\right)-A p_{0}\right\rangle \leq 0 .
$$

Analogously, we can repeat all the reasoning used to deduce (68) with $\tau(n)$ instead of $n$ and obtain

$$
\left\|x_{\tau(n)+1}-p_{0}\right\|^{2} \leq\left(1-s_{\tau(n)}\right)\left\|x_{\tau(n)}-p_{0}\right\|^{2}+s_{\tau(n)} \sigma_{\tau(n)},
$$

where

$$
\begin{aligned}
s_{\tau(n)} & =1-\left(1-\alpha_{\tau(n)} \bar{\gamma}\right)-2 \alpha_{\tau(n)} \rho, \\
\sigma_{\tau(n)} & =\frac{\alpha_{\tau(n)}}{s_{\tau(n)}}\left[2\left\langle U_{\tau(n)} x_{\tau(n)}-x_{\tau(n)}, f\left(x_{\tau(n)}\right)-A p_{0}\right\rangle\right. \\
+ & \left.2\left\langle x_{\tau(n)}-p_{0}, f\left(p_{0}\right)-A p_{0}\right\rangle\right]+\alpha_{n} O(1),
\end{aligned}
$$


So, from Xu's Lemma, taking in account (74) and (75), we have

$$
\left\|x_{\tau(n)}-p_{0}\right\|^{2} \longrightarrow 0
$$

Once again by (44) we deduce $x_{n} \rightarrow p_{0}$.

Remark 9. Theorem 1.1 in [2] is a corollary of our main result, but the technique of proof is completely different. Here we used the strong quasinonexpansivity of the mapping $S$. In Theorem 1 we used the following properties of a type average $S_{\delta}=(1-\delta) I+\delta S$ of a nonspreading mapping $S$ :

$$
\begin{aligned}
& \text { (i) }\left\|S_{\delta} x-S_{\delta} y\right\|^{2} \leq\|x-y\|^{2}+(2 / \delta)\left\langle x-S_{\delta} x, y-S_{\delta} y\right\rangle- \\
& \quad(1-\delta)\left\|\left(x-S_{\delta} x\right)-\left(S_{\delta} y\right)\right\|^{2} . \\
& \text { (ii) }(\delta / 2)\|x-S x\|^{2} \leq\left\langle x-S_{\delta} x, x-q\right\rangle, \forall q \in \operatorname{Fix}(S) .
\end{aligned}
$$

From Proposition 3 we know that there exist nonspreading mappings that are not strongly quasinonexpansive mappings.

So we do not know if the main result remains true if we take a nonspreading mapping $S$ instead of a strongly quasinonexpansive mapping, or of type average $S_{\delta}$ of a nonspreading mapping.

The question remains open. However we would like to note that, in all the examples that we know, the algorithm works also with nonspreading mappings.

\section{Conflict of Interests}

The authors declare that they have no competing interests.

\section{Authors' Contribution}

All authors contributed equally and significantly in writing this paper. All authors read and approved the final paper.

\section{Acknowledgment}

This project was funded by the Deanship of Scientific Research (DSR), King Abdulaziz University, under Grant no. 29-130-35-HiCi. The authors, therefore, acknowledge with thanks technical and financial support of KAU.

\section{References}

[1] S. Iemoto and W. Takahashi, "Approximating common fixed points of nonexpansive mappings and nonspreading mappings in a Hilbert space," Nonlinear Analysis: Theory, Methods \& Applications, vol. 71, no. 12, pp. e2082-e2089, 2009.

[2] N. Hussain, G. Marino, and A. A. N. Abdou, "On Mann's method with viscosity for nonexpansive and nonspreading mappings in Hilbert spaces," Abstract and Applied Analysis, vol. 2014, Article ID 152530, 11 pages, 2014.

[3] K. Aoyama, S. Iemoto, F. Kohsaka, and W. Takahashi, "Fixed point and ergodic theorems for $\lambda$-hybrid mappings in Hilbert spaces," Journal of Nonlinear and Convex Analysis, vol. 11, no. 2, pp. 335-343, 2010.

[4] S. Itoh and W. Takahashi, "The common fixed point theory of singlevalued mappings and multi-valued mappings," Pacific Journal of Mathematics, vol. 79, no. 2, pp. 493-508, 1978.
[5] K. Aoyama and F. Kohsaka, "Fixed point and mean convergence theorems for a family of lambda-hybrid mappings," Journal of Nonlinear Analysis and Optimization: Theory \& Applications, vol. 2, no. 1, pp. 87-95, 2011.

[6] K. Wongchan and S. Saejung, "On the strong convergence of viscosity approximation process for quasinonexpansive mappings in Hilbert spaces," Abstract and Applied Analysis, vol. 2011, Article ID 385843, 9 pages, 2011.

[7] R. E. Bruck and S. Reich, "Nonexpansive projections and resolvents of accretive operators in Banach spaces," Houston Journal of Mathematics, vol. 3, no. 4, pp. 459-470, 1977.

[8] S. Saejung, "Halpern's iteration in Banach spaces," Nonlinear Analysis: Theory, Methods \& Applications, vol. 73, no. 10, pp. 3431-3439, 2010.

[9] G. Marino and H.-K. Xu, "A general iterative method for nonexpansive mappings in Hilbert spaces," Journal of Mathematical Analysis and Applications, vol. 318, no. 1, pp. 43-52, 2006.

[10] H.-K. Xu, "Iterative algorithms for nonlinear operators," Journal of the London Mathematical Society, vol. 66, no. 1, pp. 240-256, 2002.

[11] P.-E. Mainge, "Strong convergence of projected subgradient methods for nonsmooth and nonstrictly convex minimization," Set-Valued Analysis, vol. 16, no. 7-8, pp. 899-912, 2008. 


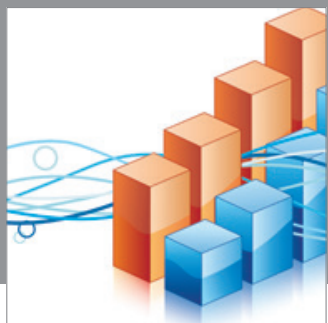

Advances in

Operations Research

mansans

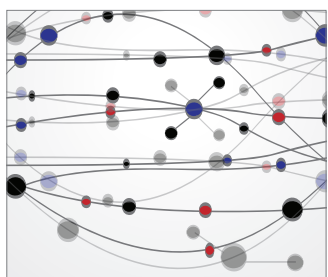

The Scientific World Journal
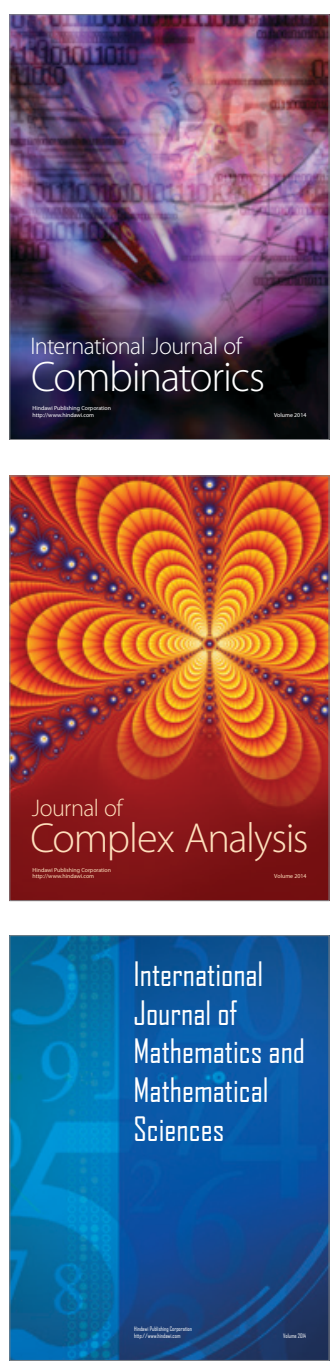
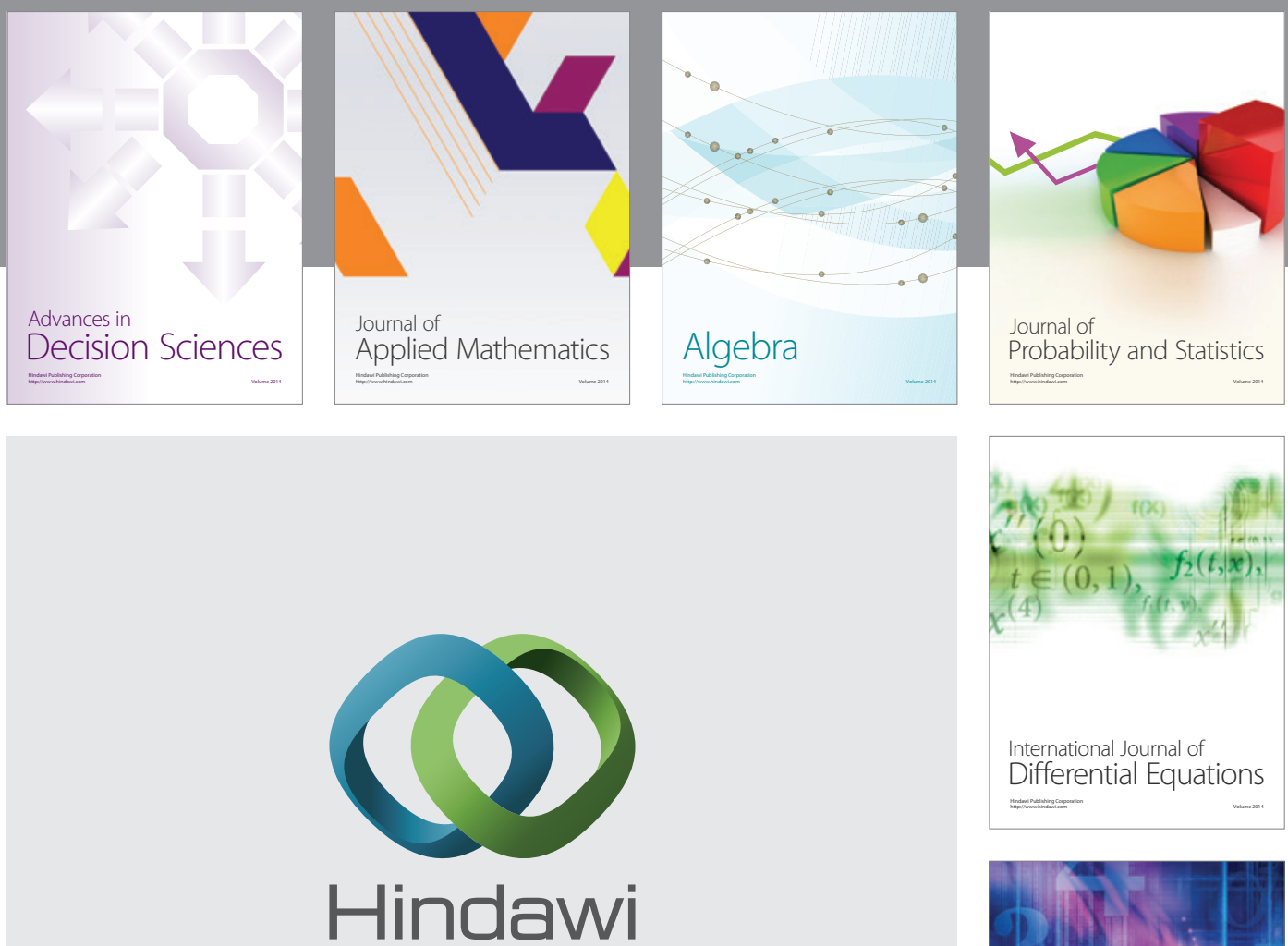

Submit your manuscripts at http://www.hindawi.com
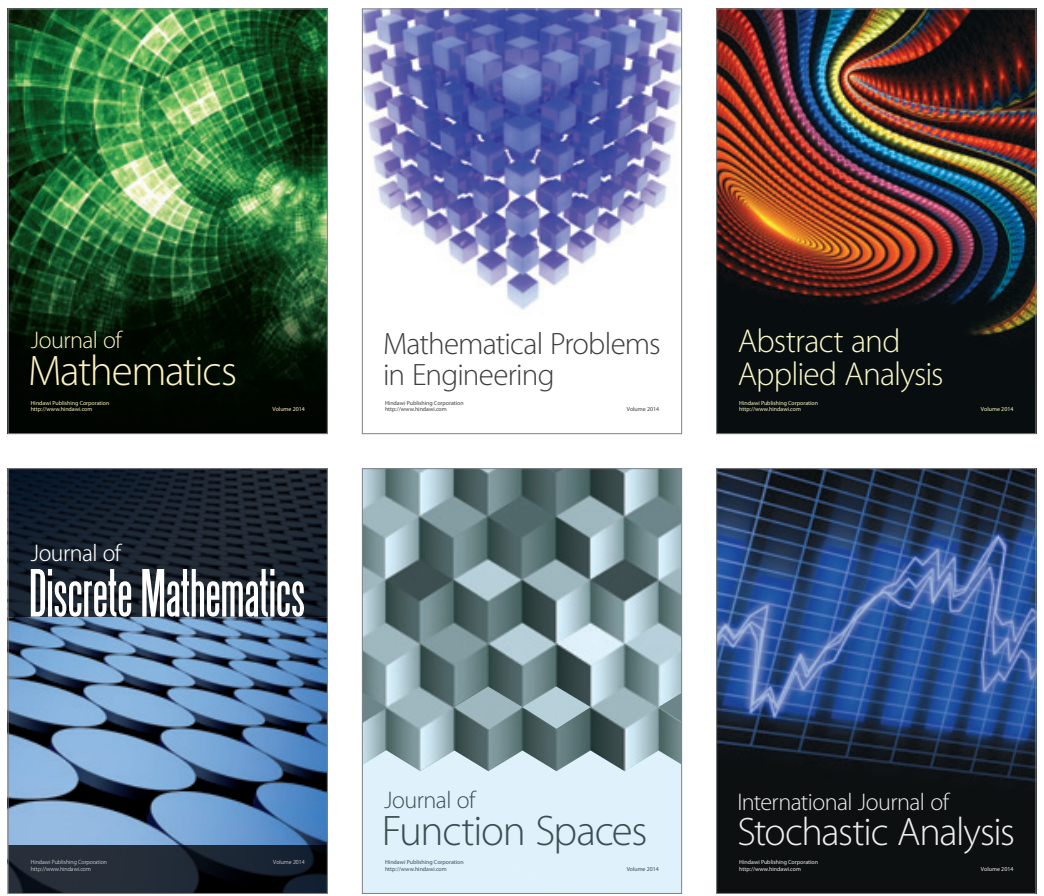

Journal of

Function Spaces

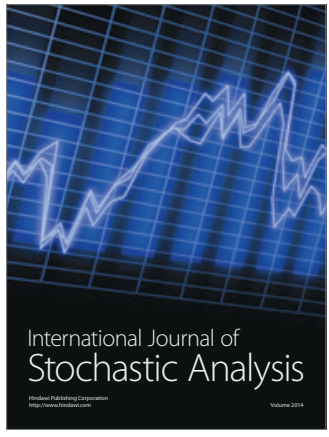

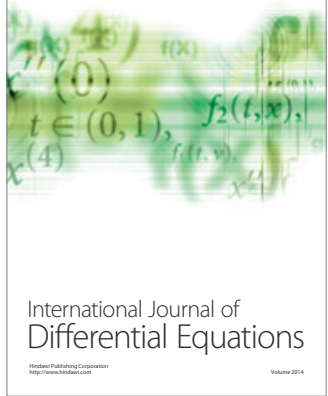
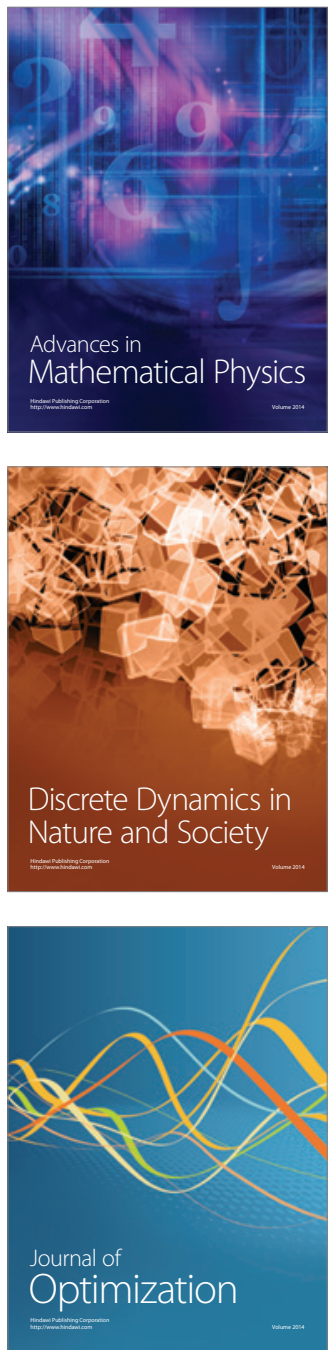